\title{
Diketopiperazines from the Cordyceps-Colonizing Fungus Epicoccum
}

\section{nigrum}

Huijuan Guo, ${ }^{\dagger}$ Bingda Sun,${ }^{\dagger}$ Hao Gao,${ }^{\ddagger}$ Xulin Chen,${ }^{\S}$ Shuchun Liu, ${ }^{\dagger}$ Xinsheng Yao, ${ }^{\ddagger}$ Xingzhong Liu, ${ }^{\dagger}$ and Yongsheng Che*, ${ }^{\dagger}$

Key Laboratory of Systematic Mycology \& Lichenology, Institute of Microbiology, Chinese Academy of Sciences, Beijing 100190, People's Republic of China, Institute of Traditional Chinese Medicine and Natural Products, College of Pharmacy, Jinan University, Guangzhou 510632, People's Republic of China, and State Key Laboratory of Virology, Wuhan Institute of Virology, Chinese Academy of Sciences, Wuhan 430071, People's Republic of China

\section{Contents}

\section{Page}

1) Figure S1. ${ }^{1} \mathrm{H}$ NMR spectrum of epicoccin E (1; $\left.500 \mathrm{MHz}, \mathrm{DMSO}-d_{6}\right)$

2) Figure S2. ${ }^{13} \mathrm{C}$ NMR spectrum of epicoccin E $\left(\mathbf{1} ; 100 \mathrm{MHz}, \mathrm{DMSO}-d_{6}\right)$

3) Figure S3. ${ }^{1} \mathrm{H}$ NMR spectrum of epicoccin F (2; $\left.500 \mathrm{MHz}, \mathrm{DMSO}-d_{6}\right)$

4) Figure S4. ${ }^{13} \mathrm{C}$ NMR spectrum of epicoccin F (2; $100 \mathrm{MHz}$, DMSO- $\left.d_{6}\right)$

5) Figure S5. ${ }^{1} \mathrm{H}$ NMR spectrum of epicoccin G (3; $\left.500 \mathrm{MHz}, \mathrm{DMSO}-d_{6}\right)$

6) Figure S6. ${ }^{13} \mathrm{C}$ NMR spectrum of epicoccin G $\left(3 ; 100 \mathrm{MHz}, \mathrm{DMSO}-d_{6}\right)$

7) Figure S7. ${ }^{1} \mathrm{H}$ NMR spectrum of epicoccin $\mathrm{H}\left(\mathbf{4} ; 500 \mathrm{MHz}, \mathrm{DMSO}-d_{6}\right) \quad 8$

8) Figure S8. ${ }^{13} \mathrm{C}$ NMR spectrum of epicoccin $\mathrm{H}\left(\mathbf{4} ; 100 \mathrm{MHz}, \mathrm{DMSO}-d_{6}\right)$

9) Figure S9. ${ }^{1} \mathrm{H}$ NMR spectrum of diphenylalazine A (5; $\left.400 \mathrm{MHz}, \mathrm{DMSO}-d_{6}\right) \quad 10$

10) Figure S10. ${ }^{13} \mathrm{C}$ NMR spectrum of diphenylalazine A $\left(5 ; 100 \mathrm{MHz}, \mathrm{DMSO}-d_{6}\right) 11$

11) Figure S11. ${ }^{1} \mathrm{H}$ NMR spectrum of diphenylalazine B (6; $\left.400 \mathrm{MHz}, \mathrm{DMSO}-d_{6}\right) 12$

12) Figure S12. ${ }^{13} \mathrm{C}$ NMR spectrum of diphenylalazine B $\left(\boldsymbol{6} ; 100 \mathrm{MHz}, \mathrm{DMSO}-d_{6}\right) 13$

13) Figure S13. CD spectra of 1 and 2 in $\mathrm{MeOH}$ 
* To whom correspondence should be addressed. Tel./Fax: +86 10 82618785. E-mail: cheys@im.ac.cn.

$\dagger$ Institute of Microbiology.

‡ Jinan University.

$\S$ Wuhan Institute of Virology. 
Figure S1. ${ }^{1} \mathrm{H}$ NMR Spectrum of Epicoccin E $\left(\mathbf{1} ; 500 \mathrm{MHz}\right.$, DMSO- $\left.d_{6}\right)$

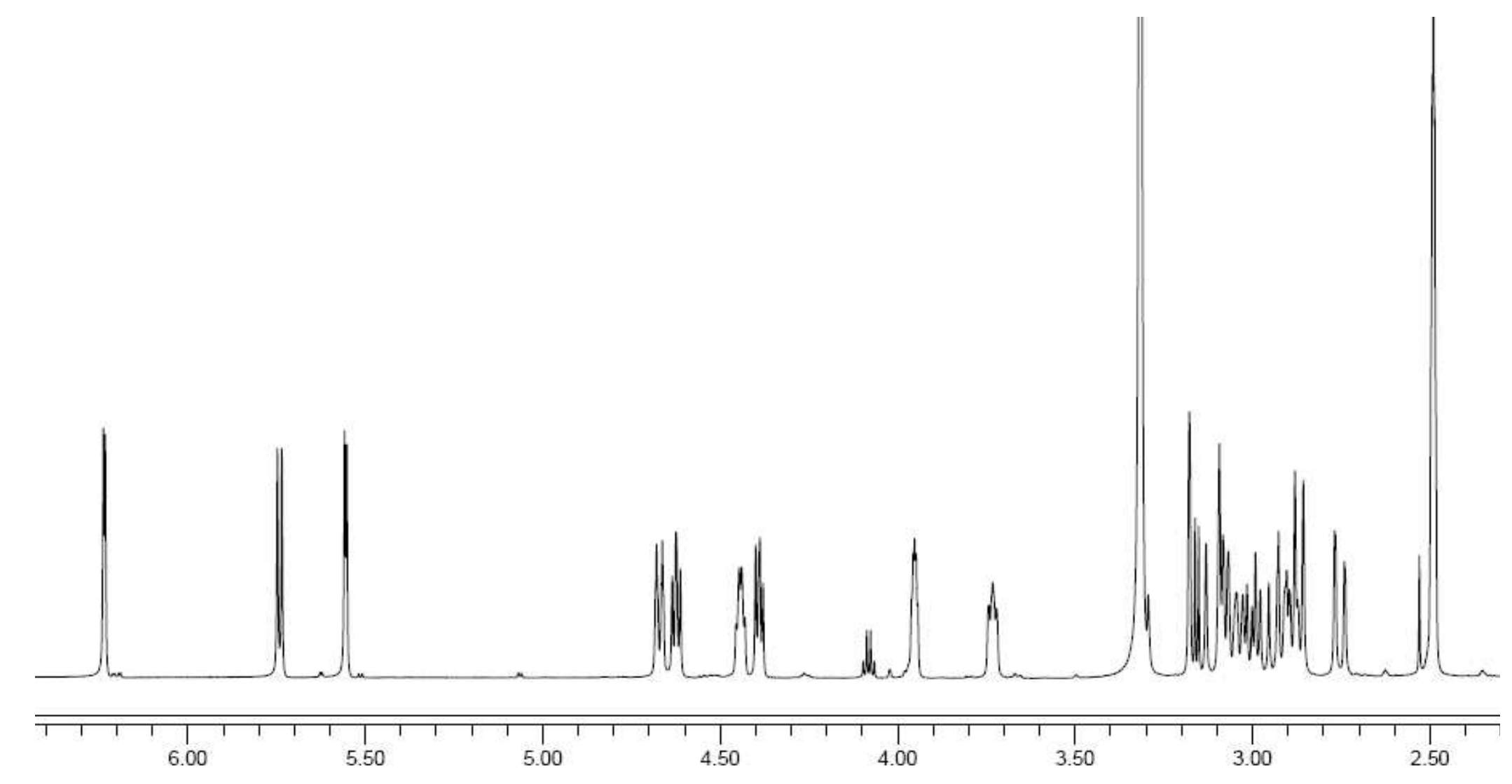


Figure S2. ${ }^{13} \mathrm{C}$ NMR Spectrum of Epicoccin E $\left(\mathbf{1} ; 100 \mathrm{MHz}\right.$, DMSO- $\left.d_{6}\right)$

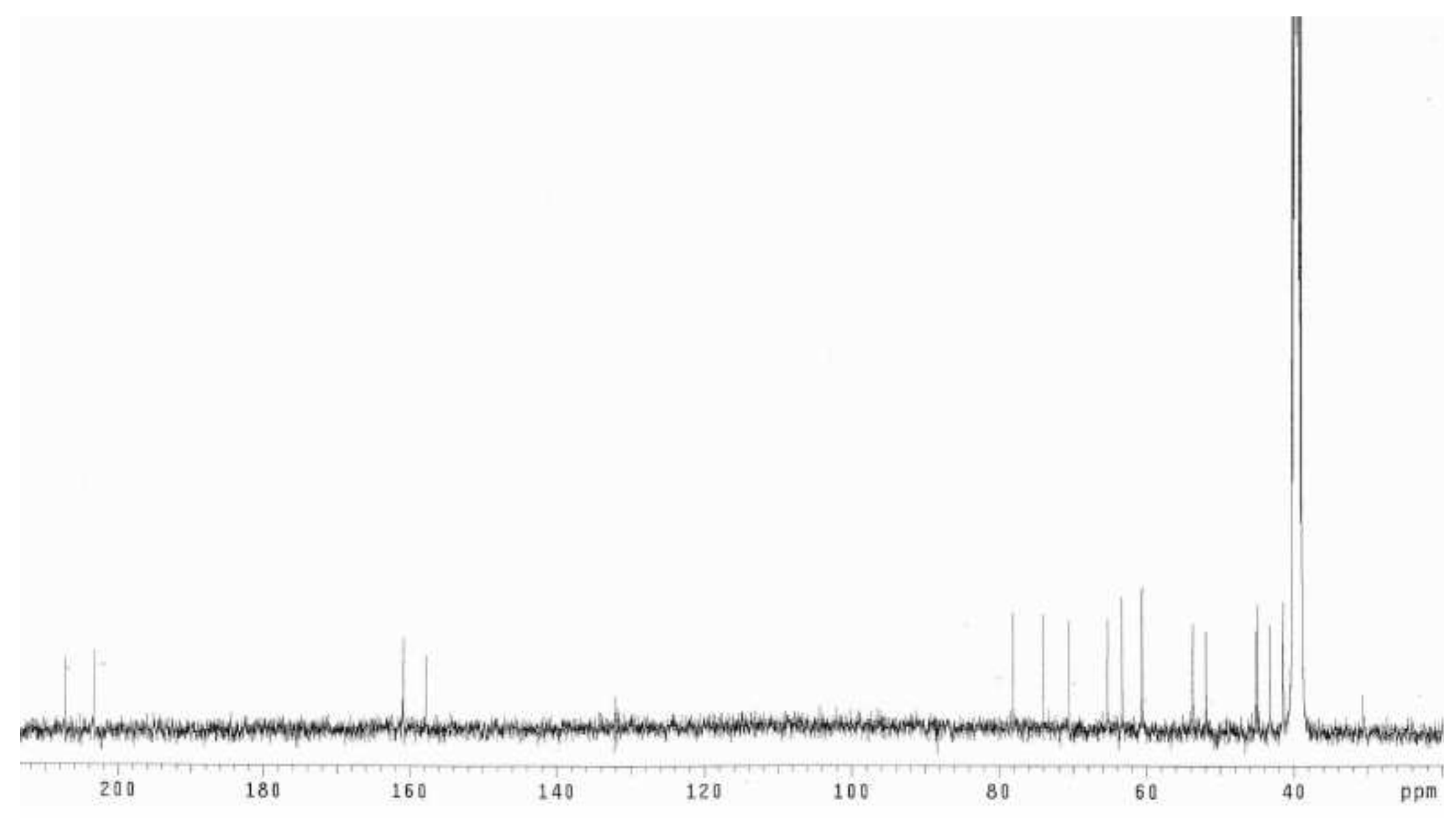


Figure S3. ${ }^{1} \mathrm{H}$ NMR Spectrum of Epicoccin F $\left(2 ; 500 \mathrm{MHz}\right.$, DMSO- $\left.d_{6}\right)$

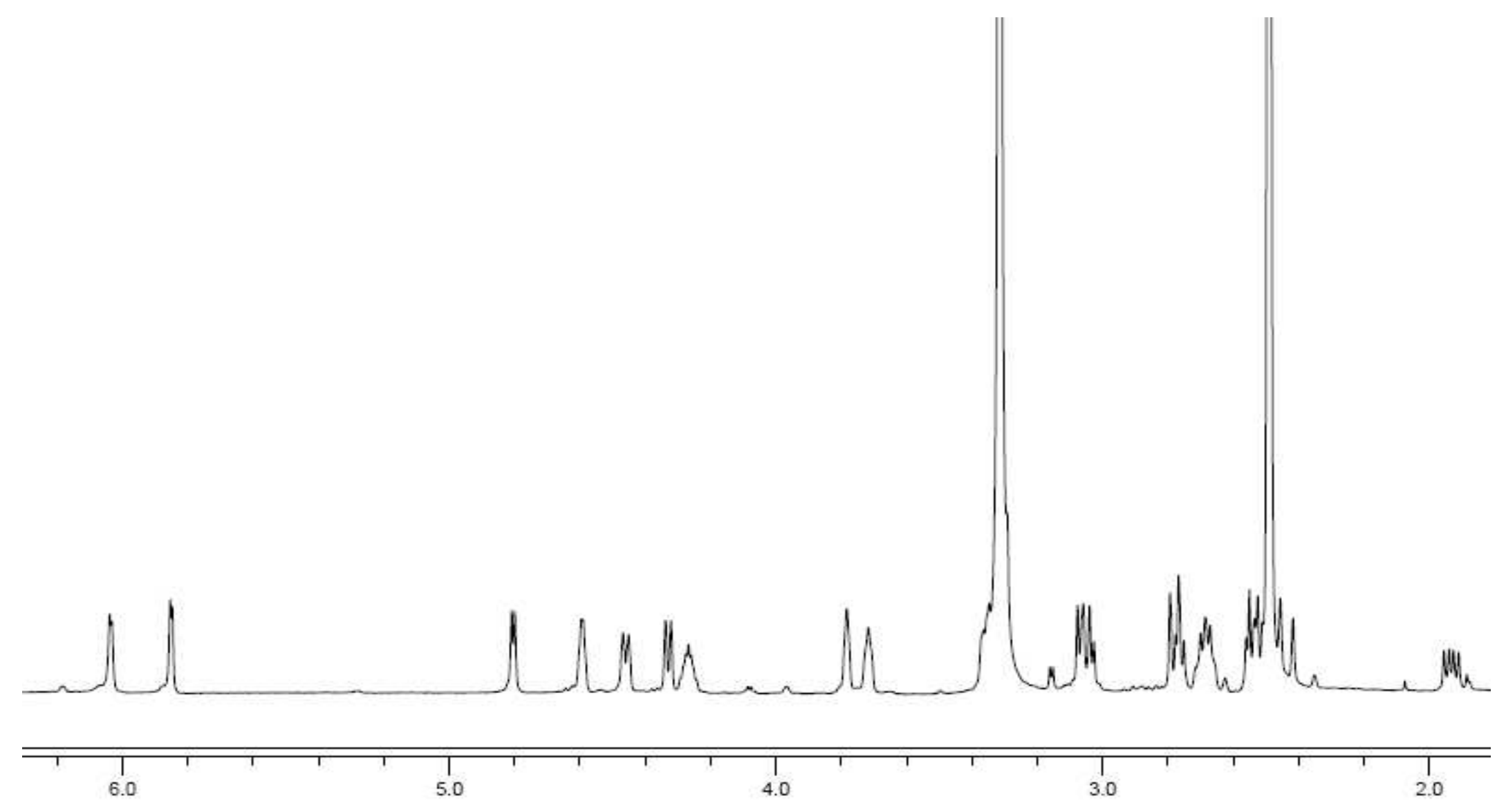


Figure S4. ${ }^{13} \mathrm{C}$ NMR Spectrum of Epicoccin F $\left(2 ; 100 \mathrm{MHz}\right.$, DMSO- $\left.d_{6}\right)$

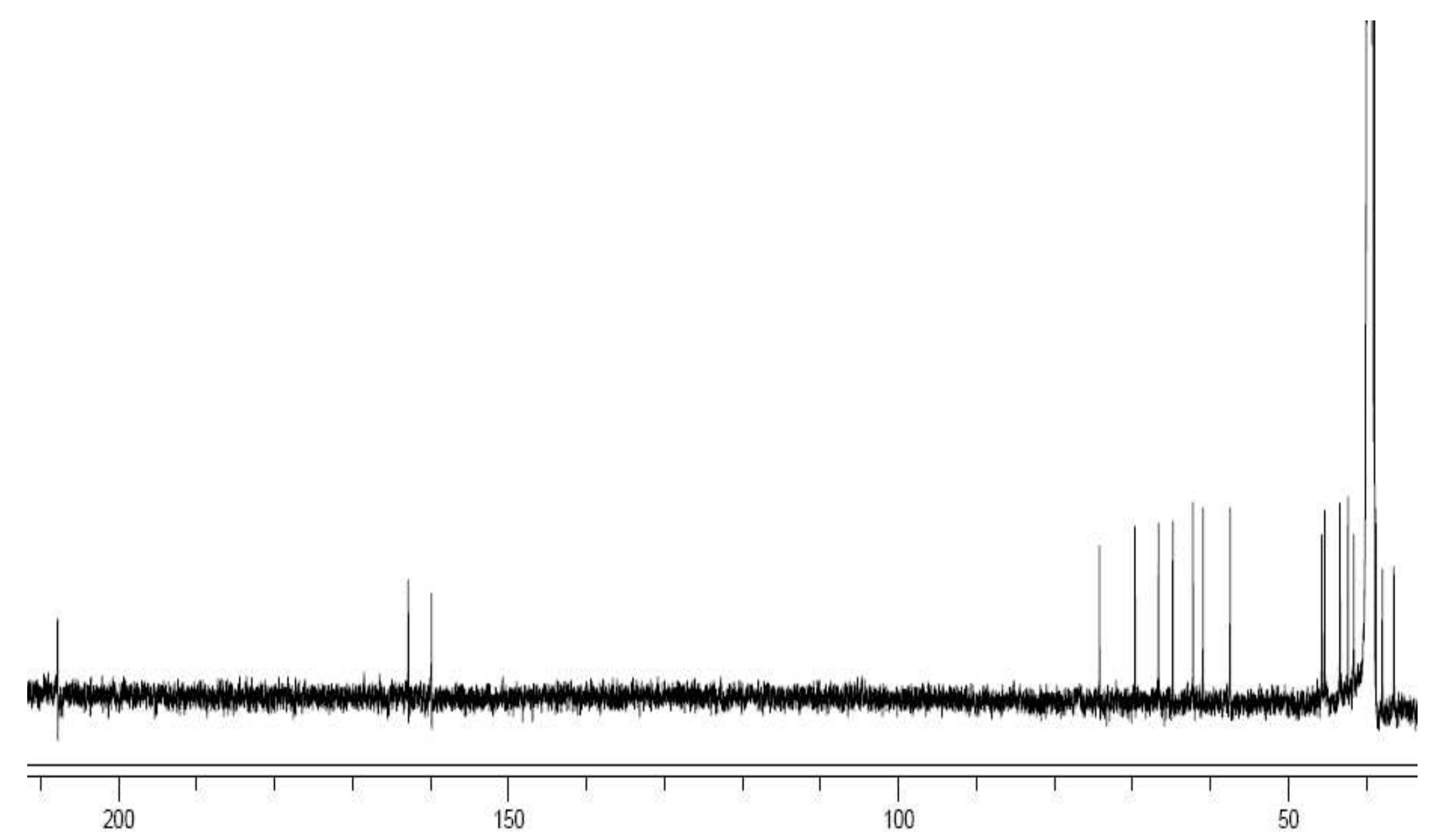


Figure S5. ${ }^{1} \mathrm{H}$ NMR Spectrum of Epicoccin G $\left(3 ; 500 \mathrm{MHz}\right.$, DMSO- $\left.d_{6}\right)$

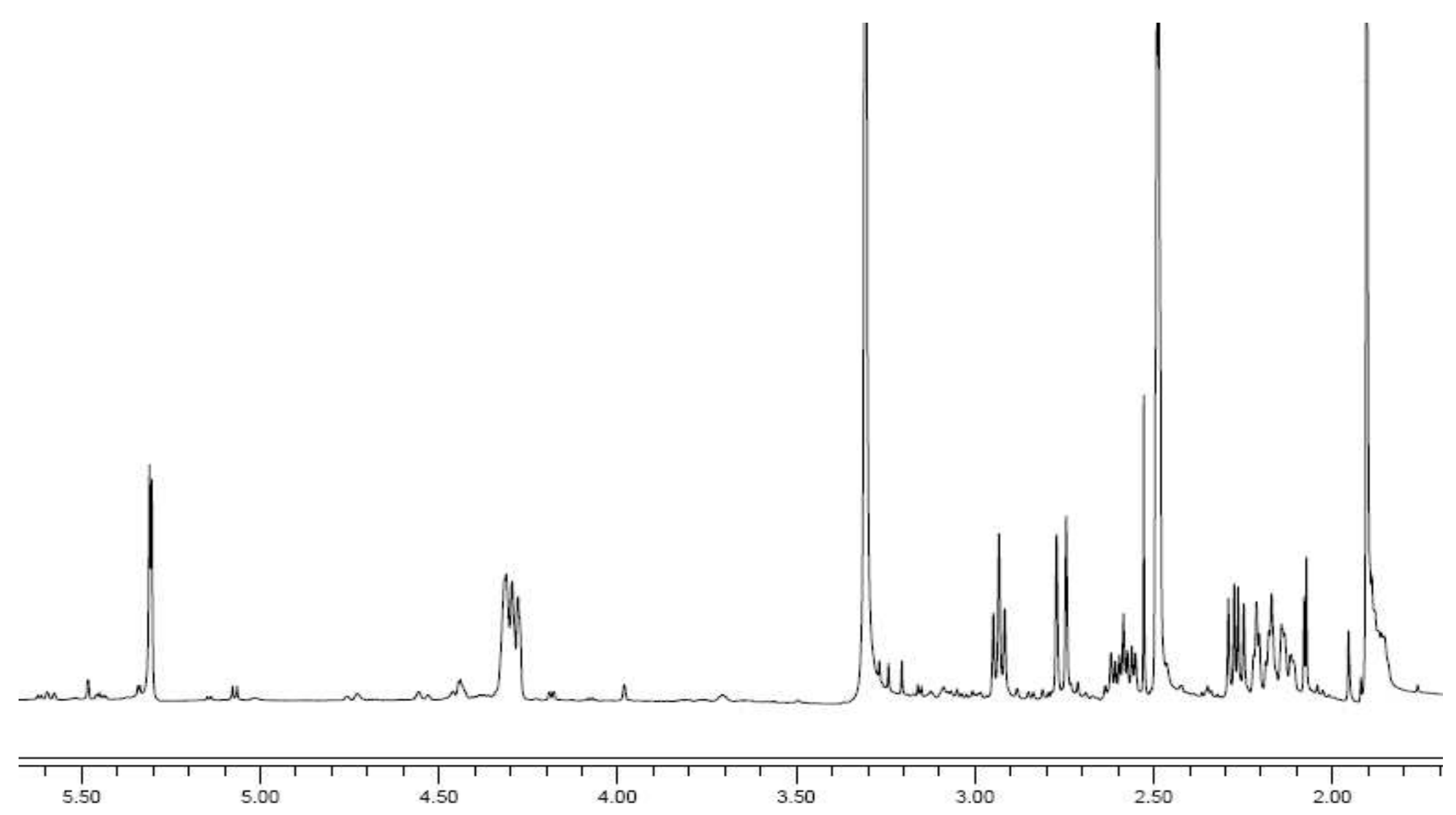


Figure S6. ${ }^{13} \mathrm{C}$ NMR Spectrum of Epicoccin G $\left(\mathbf{3} ; 100 \mathrm{MHz}\right.$, DMSO- $\left.d_{6}\right)$

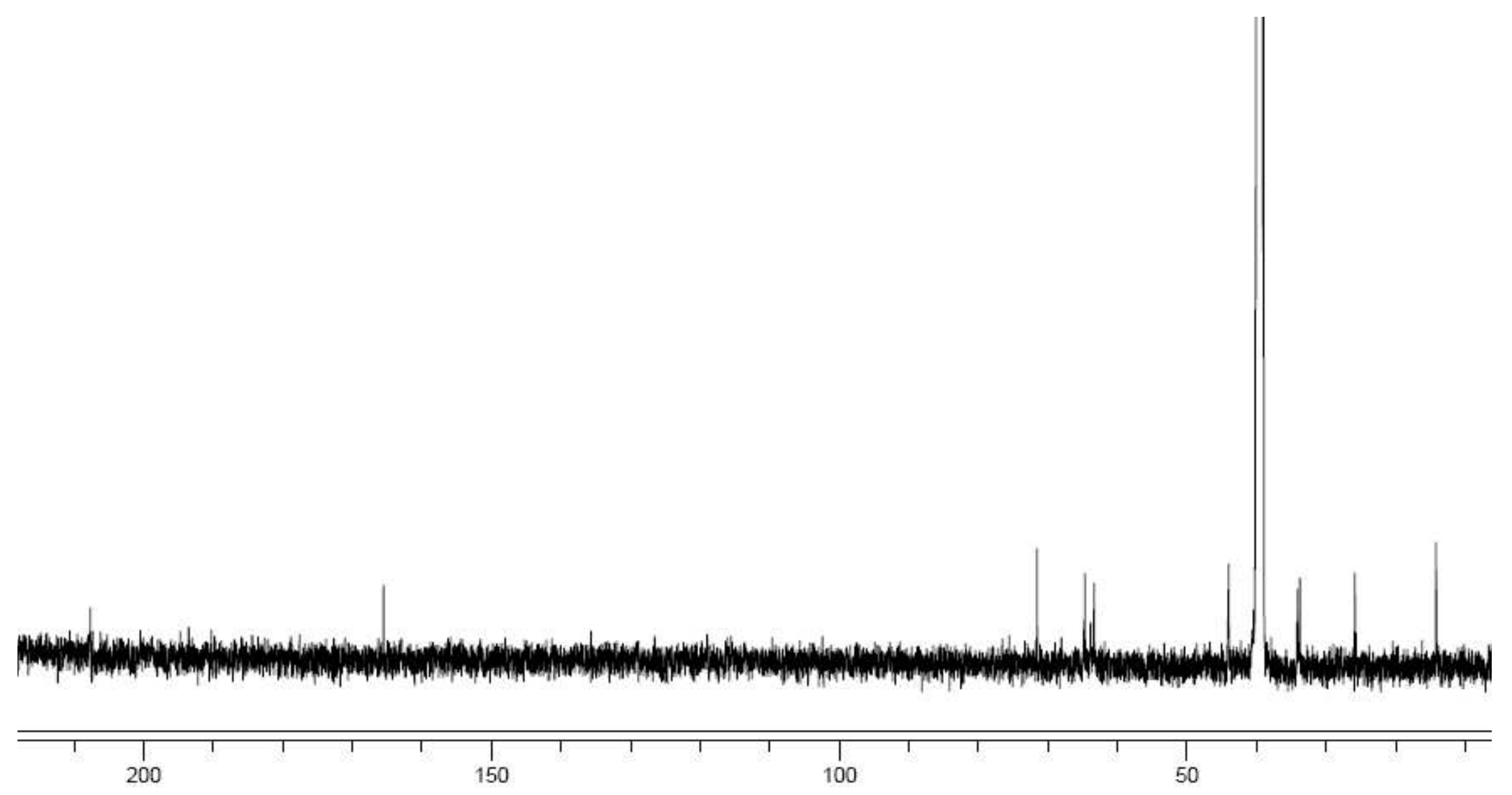


Figure S7. ${ }^{1} \mathrm{H}$ NMR Spectrum of Epicoccin H $\left(4 ; 500 \mathrm{MHz}\right.$, DMSO- $\left.d_{6}\right)$

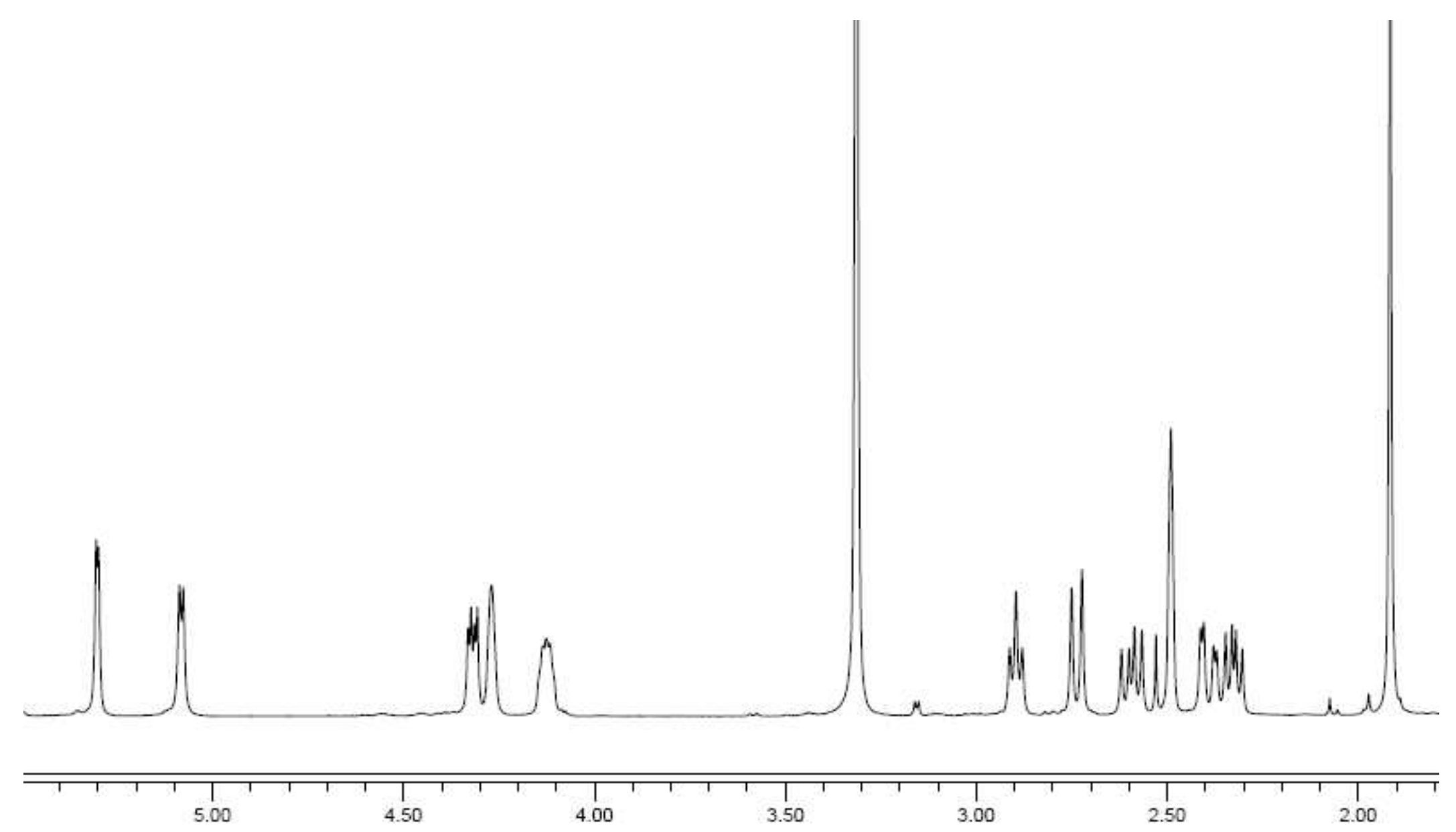


Figure S8. ${ }^{13} \mathrm{C}$ NMR Spectrum of Epicoccin H $\left(4 ; 100 \mathrm{MHz}\right.$, DMSO- $\left.d_{6}\right)$

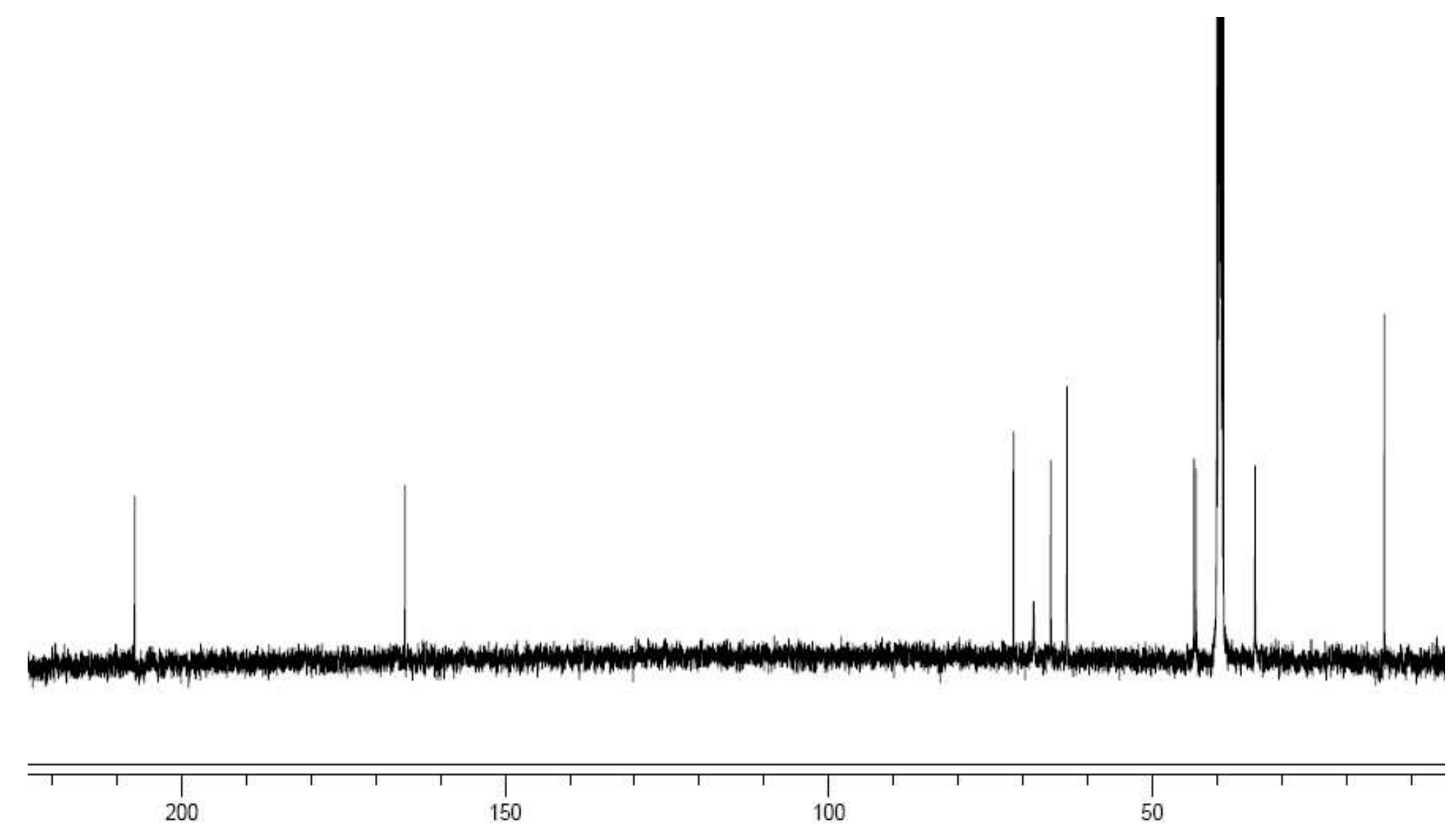


Figure S9. ${ }^{1} \mathrm{H}$ NMR Spectrum of Diphenylalazine A (5; $400 \mathrm{MHz}$, DMSO- $\left.d_{6}\right)$

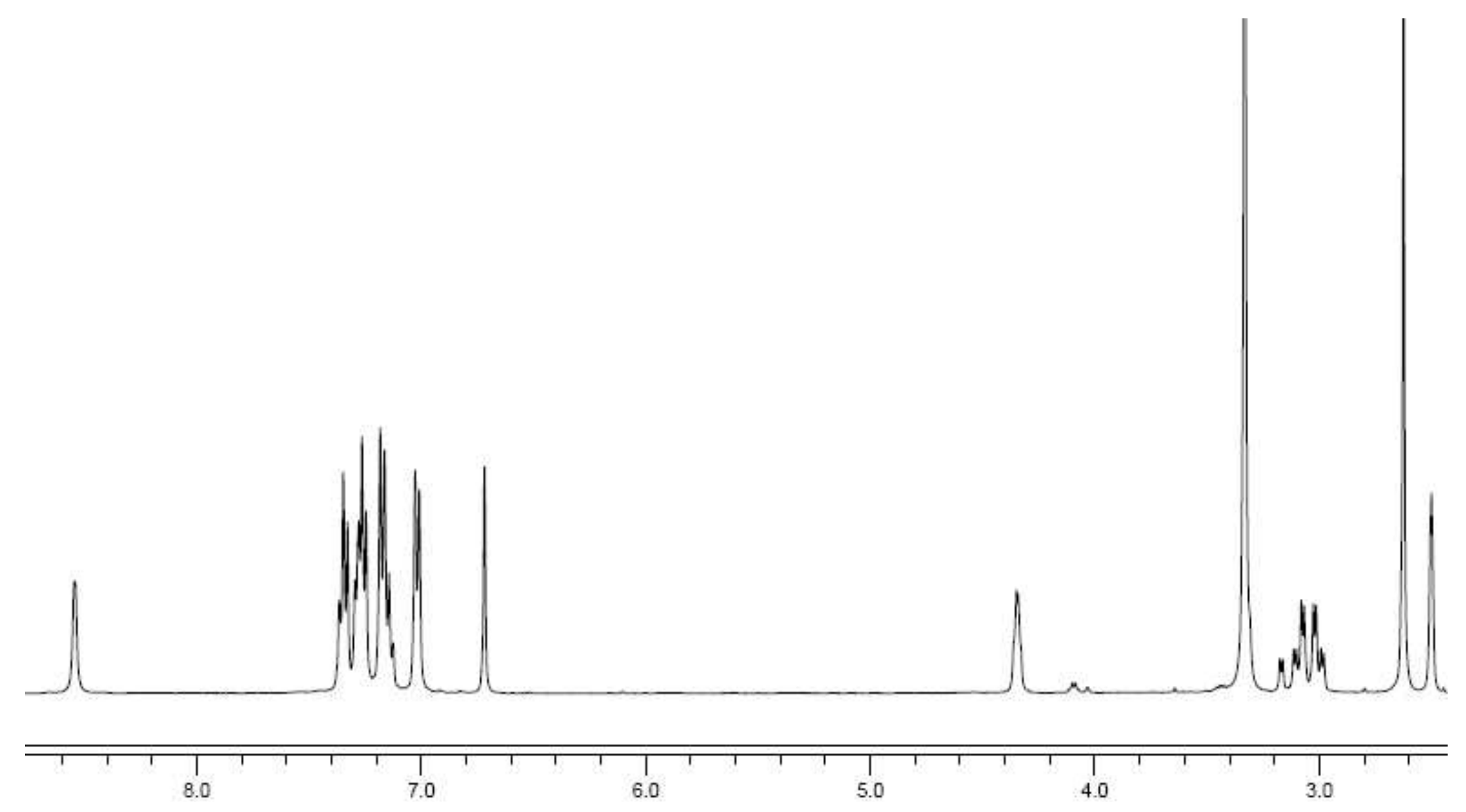


Figure S10. ${ }^{13} \mathrm{C}$ NMR Spectrum of Diphenylalazine A (5; $100 \mathrm{MHz}$, DMSO- $\left.d_{6}\right)$

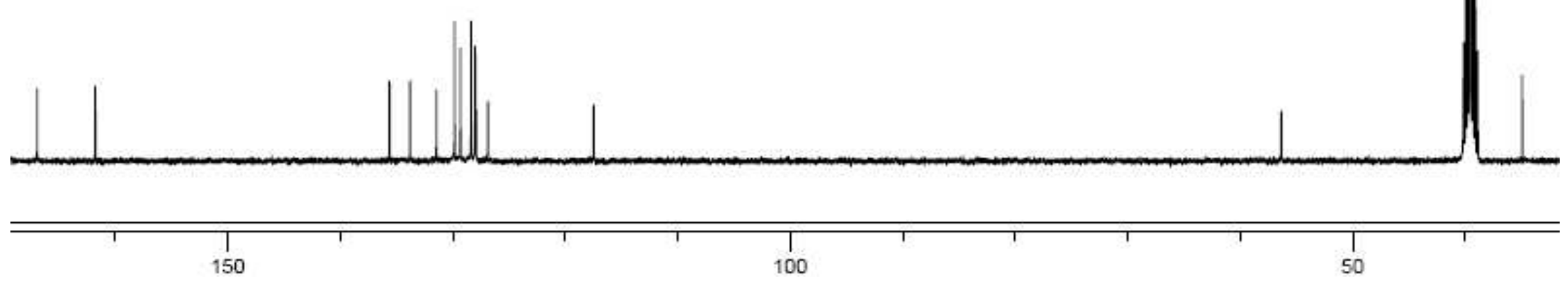


Figure S11. ${ }^{1} \mathrm{H}$ NMR Spectrum of Diphenylalazine B $\left(\mathbf{6} ; 400 \mathrm{MHz}\right.$, DMSO- $\left.d_{6}\right)$

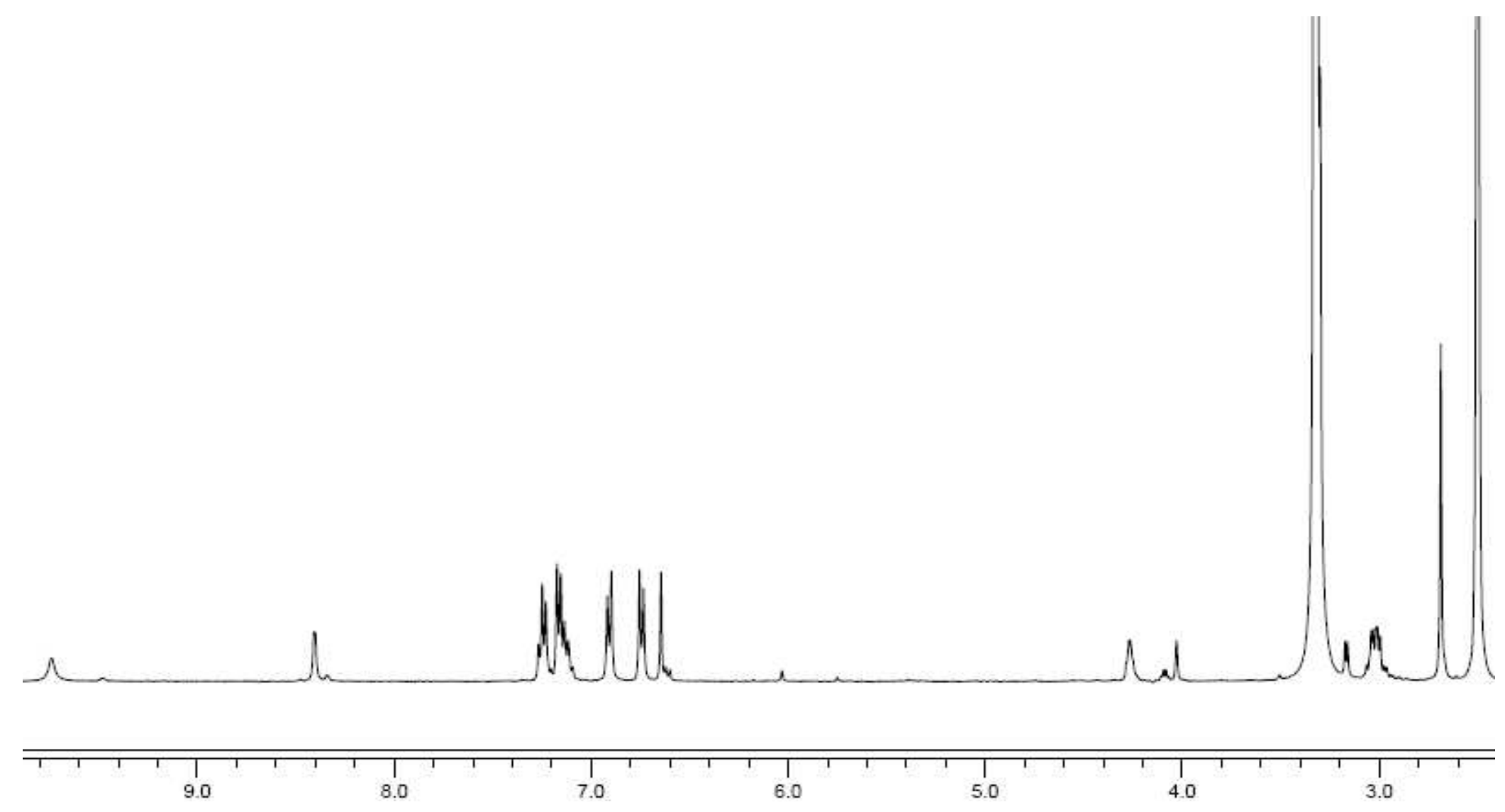


Figure S12. ${ }^{13} \mathrm{C}$ NMR Spectrum of Diphenylalazine B $\left(\mathbf{6} ; 100 \mathrm{MHz}\right.$, DMSO- $\left.d_{6}\right)$

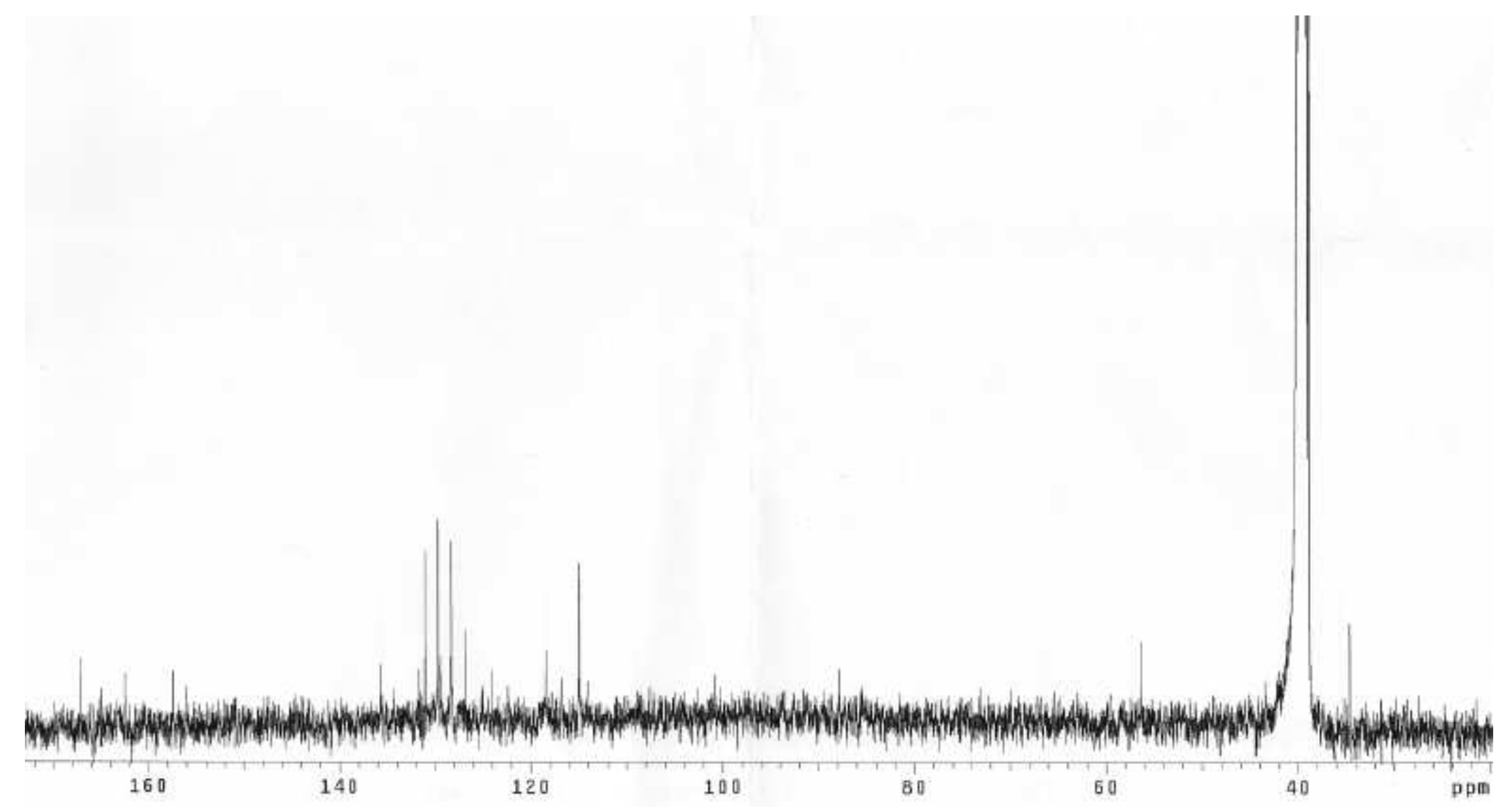


Figure S13. CD Spectra of $\mathbf{1}$ and $\mathbf{2}$ in $\mathrm{MeOH}$

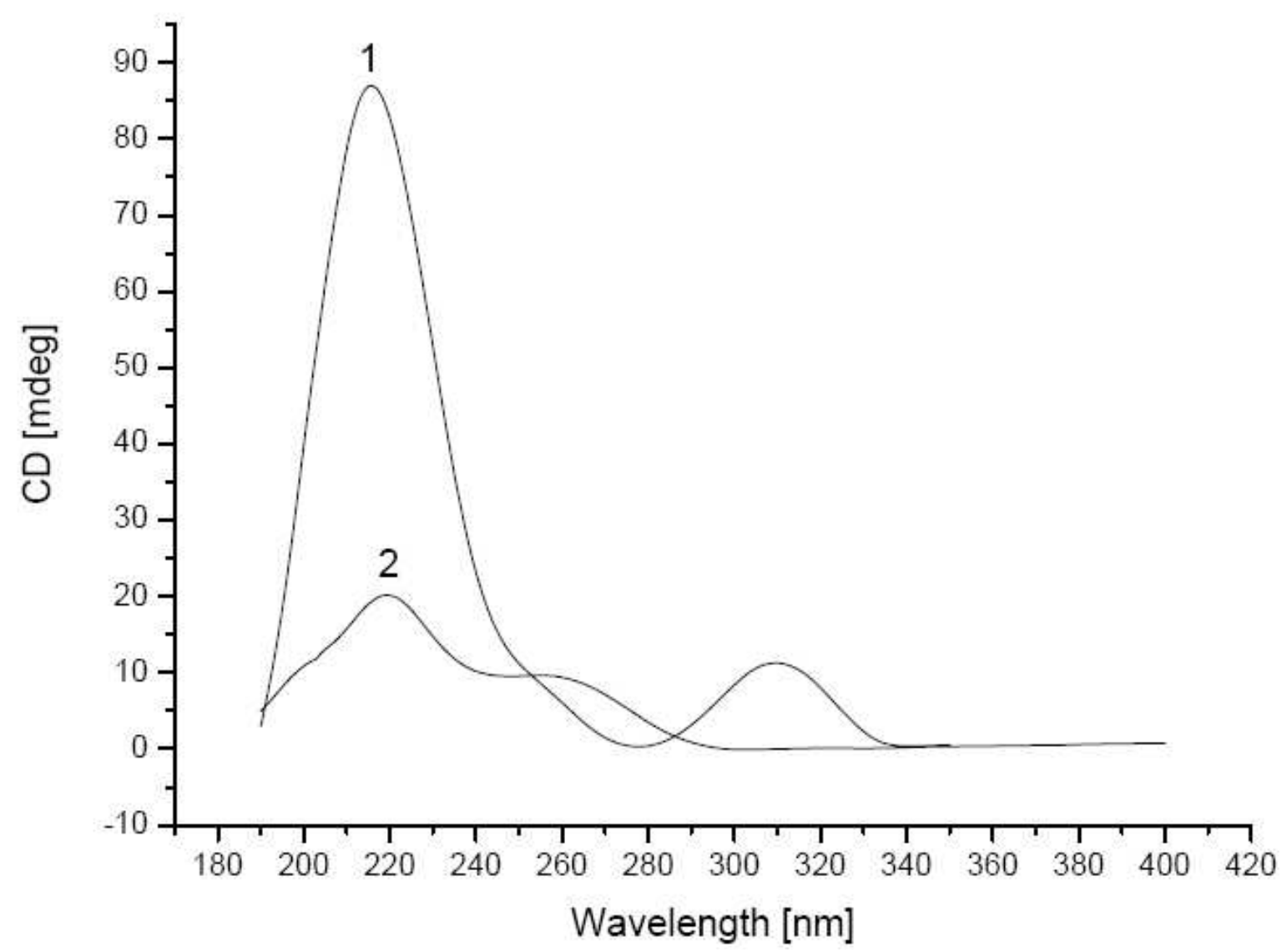

